\title{
Effects of electroacupuncture on recent stroke inpatients with incomplete bladder emptying: a preliminary study
}

\author{
Kuo-Wei Yu ${ }^{1, *}$ \\ Chien-Lin Lin ${ }^{1,2}$ \\ Chun-Chuang Hung ${ }^{3}$ \\ Eric Chieh-Lung $\mathrm{Chou}^{4}$ \\ Yueh-Ling $\mathrm{Hsieh}^{5}$ \\ Te-Mao Li ${ }^{2,3, *}$ \\ Li-Wei Chou ${ }^{1,2,6}$ \\ 'Department of Physical Medicine \\ and Rehabilitation, China Medical \\ University Hospital, Taichung, \\ Taiwan; ${ }^{2}$ School of Chinese Medicine, \\ College of Chinese Medicine, China \\ Medical University, Taichung, Taiwan; \\ ${ }^{3}$ Graduate Institute of Acupuncture \\ Science, China Medical University, \\ Taichung, Taiwan; ${ }^{4}$ Department of \\ Urology, China Medical University \\ Hospital, Taichung, Taiwan; \\ ${ }^{5}$ Department of Physical Therapy, \\ China Medical University, Taichung, \\ Taiwan; ${ }^{6}$ Acupuncture Research \\ Center, China Medical University, \\ Taichung, Taiwan \\ *These authors contributed equally \\ to this work
}

\author{
This article was published in the following Dove Press journal: \\ Clinical Interventions in Aging \\ 7 November 2012 \\ Number of times this article has been viewed
}

\begin{abstract}
Background: Incomplete bladder emptying (IBE) is defined as having a postvoid residual (PVR) urine volume greater than $100 \mathrm{~mL}$ for 2 consecutive days. IBE is common in stroke patients and could necessitate indwelling or intermittent catheterization. The condition is correlated with urinary tract infections, which could impede rehabilitation progress and increase medical costs. Treatment for patients with IBE includes bladder retraining, biofeedback, medication, and botulinum toxin injection, but none of these interventions are completely effective.

Methods: All patients with acute stroke who were admitted to the rehabilitation ward between August 2010 and April 2011 were included in the study and their PVR urine volume was checked. Electroacupuncture (EA; $1 \mathrm{~Hz}, 15$ minutes) was performed on the acupoints Sanyinjiao (SP6), Ciliao (BL32), and Pangguangshu (BL28) of stroke patients with IBE for a total of ten treatments (five times a week for 2 weeks). Bladder diaries, which included the spontaneous voiding and PVR urine volumes, were recorded during the course of treatment.
\end{abstract}

Results: The presence of IBE was not related to sex, history of diabetes mellitus, stroke type (hemorrhagic or ischemic), or stroke location $(P>0.05)$. Among the 49 patients in the study, nine $(18 \%)$ had IBE, and seven of the stroke patients with IBE were treated with EA. Increased spontaneous voiding volume and decreased PVR urine volume were noted after ten sessions of EA.

Conclusion: EA may have beneficial effects on stroke survivors with IBE, thereby making it a potential safe modality with which to improve urinary function.

Keywords: acupuncture, electroacupuncture, incomplete bladder emptying, rehabilitation, stroke, urinary retention

\section{Introduction}

A high prevalence $(44 \%-69 \%)$ of bladder dysfunction has been reported in stroke survivors; this includes urinary retention, incomplete bladder emptying (IBE), detrusor external sphincter dyssynergia, and bladder hyperactivity. ${ }^{1}$ Urinary retention may occur in approximately $29 \%$ of the stroke patients in rehabilitation wards. ${ }^{2}$ Catheterization is commonly performed to manage episodes of acute urinary retention in stroke survivors. However, indwelling catheters may affect rehabilitation activity or daily living, lead to urinary infection, and interfere with the reestablishment of a normal voiding pattern. ${ }^{3}$ A significantly higher rate of urinary infection has been observed in individuals with $\mathrm{IBE} ;{ }^{4}$ thus, indwelling catheters should be removed as soon as possible. The incidence of IBE is initially $56 \%$ in stroke patients, but it decreases to $32 \%$ over time. ${ }^{4}$ The use of $\alpha$-blockers may increase emptying, but side effects such as orthostatic hypotension may affect patient rehabilitation. Intermittent catheterization is another option for IBE management; ${ }^{5}$ this procedure, however, is dependent on patient ability and family support.
Correspondence: Li-Wei Chou Department of Physical Medicine and Rehabilitation, China Medical University Hospital, No 2 Yuh-Der Road, Taichung, 404, Taiwan, Republic of China Tel +886 422052121 Extn 238I Fax +886 42202604 I

Email chouliwe@tcts.seed.net.tw; chouliwe@gmail.com 
Several studies on stroke survivors have suggested that acupuncture therapy provides significant benefits for stroke patients. $^{6-11}$ Animal experiments have demonstrated that electroacupuncture (EA) could improve the bladder emptying function. ${ }^{12,13}$ Only a few high-quality studies have focused on this subject, and the lack of appropriate inclusion criteria or objective tools to assess the treatment effects of EA has been noted. One randomized trial demonstrated that the stimulation of acupuncture points could improve post-stroke urinary symptoms, but only moxibustion therapy was mentioned in their study. ${ }^{14}$ Thus, we devised this prospective study to explore the improvement of the bladder emptying function in post-stroke patients after EA treatment.

\section{Materials and methods}

\section{Patients}

From August 2010 to April 2011, patients who were transferred from the neurology or neurosurgery wards to the rehabilitation ward in the China Medical University Hospital, Taichung, Taiwan, were considered for inclusion if they were diagnosed with infarction or hemorrhagic stroke for the first time and did not suffer from an active urinary tract infection. During the study enrollment period, the patients continued to receive conventional therapy (diet treatment, hypoglycemic agents, insulin, and hypertensive agents). No major changes were made to the prescribed medication during the study.

The specific inclusion criterion at enrollment was the presence of IBE, which was defined as having a postvoid residual (PVR) urine volume greater than $100 \mathrm{~mL}$ for 2 consecutive days at the rehabilitation ward. ${ }^{15}$ Exclusion criteria included dermatitis over the acupuncture points and active urinary tract infection, as well as the prior use of anticholinergic drugs, cholinergic drugs, and $\alpha$-blockers. All patients gave their informed consent, and the Institutional Review Board of the university approved the study.

\section{Identification of acupuncture points}

Three acupuncture points, namely Sanyinjiao (SP6), Ciliao (BL32), and Pangguangshu (BL28), ${ }^{16}$ were selected for the treatment of IBE according to traditional Chinese medicine theories. In this study, the acupuncture points were identified by an experienced licensed acupuncturist, and the three acupuncture points were marked for subsequent study by the same acupuncturist using "cun". Cun is the unit of measurement used for locating acupuncture points. One cun is equal to the space between the distal interphalangeal joint and the proximal interphalangeal joint on the middle finger. SP6 is located 3 cun above the tip of the medial malleolus. BL32 is located in the second posterior sacral foramen which in the depression, midway between the posterior superior iliac spine and the spinous process of the second sacral vertebra. BL28 is located at the same level, 1.5 cun lateral to the median sacral crest (Figure 1).

\section{Treatment procedures}

The acupuncturist who had initially identified the acupuncture points likewise performed all the subsequent treatment procedures. Patients underwent treatment in a comfortable side-lying position. Prior to insertion of the acupuncture needle, the skin over the marked acupuncture point was cleaned with alcohol. Disposable acupuncture needles (Yuguang Industrial Co, Ltd, Taipei, Taiwan) (size \#30; $0.28 \mathrm{~mm}$ in diameter) with a length of 1 inch $(25.4 \mathrm{~mm})$ or 1.5 inches $(37 \mathrm{~mm})$ were used for each patient. Six of the bilateral acupuncture points, specifically the pairs of SP6, BL32, and BL28, were connected to an electrostimulator (Ching Ming model-05B, Ching Ming Medical Device, Taipei, Taiwan) that produced biphasic square-shaped pulses at a low frequency ( $1 \mathrm{~Hz}, 3 \mathrm{~mA}$ to $60 \mathrm{~mA}$ ) for 15 minutes. The treatment sessions were performed 5 days a week for 2 weeks.

\section{Measurement of treatment outcomes}

The basic clinical data of all patients were collected, including their age, sex, history of diabetes mellitus, stroke type, and stroke location. Bladder diaries were kept for each patient, which monitored parameters such as the spontaneous voiding and PVR urine volumes. These values were measured by a portable ultrasonic bladder scanner or by intermittent catheterization during the 10-day EA treatment. ${ }^{17}$

Effects of the EA treatment were presented in terms of the daily PVR urine ratio. This ratio was computed using the following equation: daily PVR urine ratio $=($ daily PVR urine volume/[daily PVR urine volume + spontaneous voiding volume]).

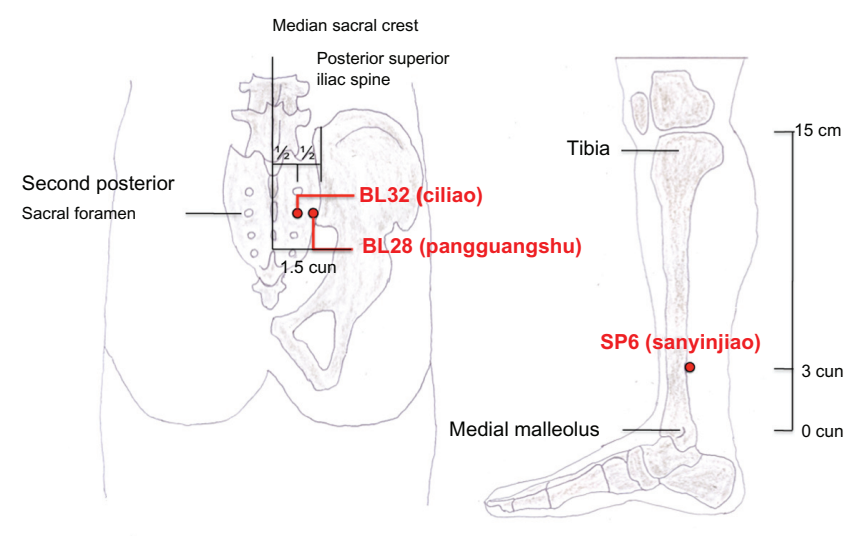

Figure I Locations of acupuncture points based on the WHO standards for the Western Pacific region. ${ }^{16}$ Redrawn by $\mathrm{Yu}$ and Chou.

Abbreviation: WHO, World Health Organization. 


\section{Statistical analyses}

The arithmetic means of all values were used for the statistical methods in this study. All analyses were performed using the SPSS version 15 software (IBM Corporation, Armonk, NY). The categorical variables were analyzed using the $\chi^{2}$-test and Fisher's exact test. Comparisons of numerical variables between patient groups were performed using the independent $t$-test. Values with $P<0.05$ were considered statistically significant.

\section{Results}

All patients with acute stroke who were admitted to the rehabilitation ward between August 2010 and April 2011 were included in this study. IBE occurred in 9 out of 49 patients (18\%). The flow diagram of the study is shown in Figure 2. For EA treatment, 7 out of the 9 eligible patients were enrolled and completed the study; their data were included in the analysis. One patient with IBE refused to participate; another withdrew due to pneumonia and was transferred to the intensive care unit.

The baseline patient characteristics are shown in Tables 1 and 2. Overall, the study population had a mean age of 65.67 years, and the majority of the participants were male. No significant associations were observed between the presence of IBE and each of the following parameters: sex, history of diabetes mellitus, stroke type (hemorrhagic or ischemic), and stroke location $(P>0.05)$.

The arithmetic means of the daily PVR urine ratio of all patients during the 10 day EA treatment are presented in Figure 3. The PVR urine ratio had the tendency to decrease as an effect of EA. IBE persisted in two of the patients after the EA treatment. A significant difference $(P<0.05)$ in the PVR urine volume of each patient was noted after the ten EA sessions.

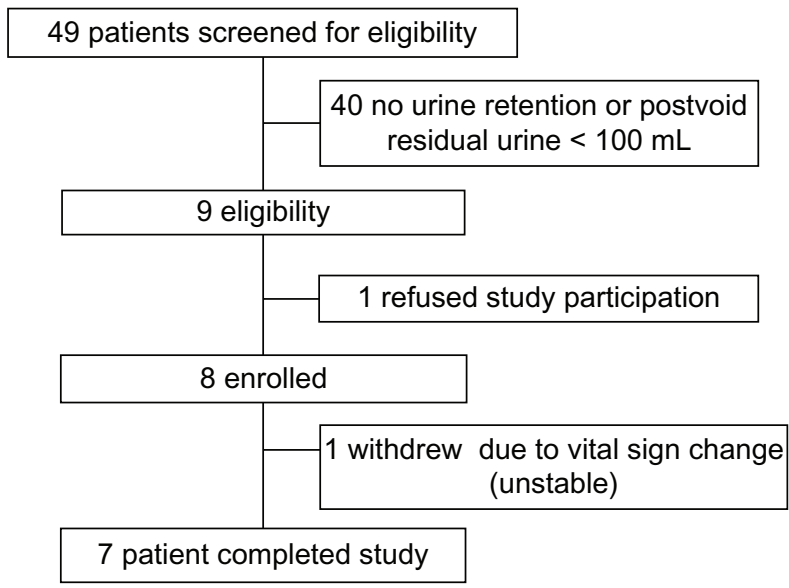

Figure 2 Flow diagram of the electroacupuncture clinical trial.
Table I Demographic information of IBE and non-IBE patients

\begin{tabular}{|c|c|c|c|}
\hline & \multicolumn{2}{|c|}{ Number of patient } & \multirow[t]{2}{*}{$P$ value } \\
\hline & IBE $(n=9)$ & Non-IBE $(n=40)$ & \\
\hline Gender & & & $>0.05$ \\
\hline Male & 6 & 23 & \\
\hline Female & 3 & 17 & \\
\hline Age & $65.67 \pm 14.58$ & $65.58 \pm 11.11$ & $>0.05$ \\
\hline Diabetes mellitus & & & $>0.05$ \\
\hline Present & 4 & 15 & \\
\hline Absent & 5 & 25 & \\
\hline Stroke type & & & $>0.05$ \\
\hline Hemorrhage & 5 & 16 & \\
\hline Infarction & 4 & 24 & \\
\hline Stroke location & & & $>0.05$ \\
\hline Cerebrum & 6 & 36 & \\
\hline Cerebellum & 2 & I & \\
\hline Brain stem & I & 3 & \\
\hline
\end{tabular}

Abbreviation: IBE, incomplete bladder emptying.

\section{Discussion Effect of EA on urinary function}

An increase in the spontaneous voiding volume and a decrease in the PVR urine volume were noted after the EA treatment. Thus, EA may have beneficial effects on stroke survivors with IBE and could be a safe modality for the improvement of urinary function.

\section{Stroke and IBE}

The two major and distinct functions of the bladder are urine storage and voiding. Burney et al reported that 28 out of 60 patients $(47 \%)$ had urinary retention within 72 hours after stroke onset. ${ }^{18}$ This high incidence rate was not surprising because the present study was performed during the acute stroke stage. The detrusor function gradually improves with time after the patient's condition has stabilized..$^{19,20}$ Kong and Young reported that urinary retention was present in $29 \%$ of patients at the subacute stage, which was observed after their admission to the rehabilitation ward within 4 weeks after stroke onset. ${ }^{2}$

In our study, $18 \%$ (9 out of 49 ) of the stroke patients who were admitted to our rehabilitation ward had IBE. After the ten EA sessions, IBE persisted in only $28 \%$ ( 2 out of 7 ) of the patients. In a previous study, Garrett found that $58 \%$ ( 28 out of 48 ) of stroke patients who were admitted to a rehabilitation ward had sustained IBE at the time of discharge. ${ }^{4}$ Another study on ischemic stroke patients showed that $17 \%$ of patients had sustained IBE at the time of discharge. ${ }^{2}$ In the present study, all ischemic stroke patients could void without IBE after the 10-day treatment course of EA. 
Table 2 Basic data of IBE patients who complete clinical trial

\begin{tabular}{llllllll}
\hline No & Sex & Age & Lesion location & Stroke type & Urodynamic study & $\begin{array}{l}\text { Pre-treatment } \\
\text { PVR }(\mathbf{m L})\end{array}$ & $\begin{array}{l}\text { Posttreatment } \\
\text { PVR*(mL) }\end{array}$ \\
\hline I & M & 82 & Cerebral cortex & Hemorrhage & Areflexia & 340 & 530 \\
2 & M & 60 & Occipital lobe & Infarction & Areflexia & 100 & 40 \\
3 & M & 59 & Cerebellum & Hemorrhage & Weak detrusor contractility & 400 & 230 \\
4 & M & 90 & Internal capsule & Infarction & Nil & 200 & 40 \\
5 & F & 69 & Thalamus & Hemorrhage & Detrusor sphincter dyynergia & 160 & 20 \\
6 & F & 71 & Cerebellum & Hemorrhage & Detrusor overactivity & 300 & 75 \\
7 & M & 40 & Medulla & Infarction & Normal detrusor function & 300 & 0 \\
\hline
\end{tabular}

Note: *Posttreatment PVR: data after 10-day treatment course of electroacupuncture.

Abbreviations: IBE, incomplete bladder emptying; PVR, postvoid residual.

\section{IBE management}

For stroke survivors with urinary retention, catheterization is the most common treatment to relieve pain and distention. However, indwelling catheters may increase urinary tract infection rates or affect rehabilitation and daily living activities. $^{3}$ Thus, indwelling catheters should be removed as soon as possible. Intermittent catheterization is an alternative solution that could improve the vesical defense mechanisms and decrease the incidence of bacteriuria. ${ }^{21}$ Moreover, intermittent catheterization could provide information on PVR urine. The actual determination of PVR urine volume after catheterization could aid in the prescription of a rational program for clean intermittent catheterization (CIC). However, a CIC program proves ineffective if the attendants and family members are unable to perform it. Other problems that were encountered with CIC included urethral trauma and occasional bladder stone formation.
The use of $\alpha$-blockers may increase bladder emptying. However, the side effect of orthostatic hypotension may affect patient rehabilitation. The oral forms of these cholinergic agents have not been proven effective at levels that could be tolerated by patients. ${ }^{22}$ Thus, EA could be an alternative method in the management of IBE.

\section{Possible mechanisms of urinary retention via acupuncture treatment}

Acupuncture is a safe form of treatment, ${ }^{23}$ and several studies have suggested its beneficial effects on the treatment of stroke survivors. ${ }^{24,25}$ One study provided evidence that acupuncture may be clinically useful for improving bladder function in diabetics. ${ }^{26}$ However, only a few high-quality studies have focused on IBE in stroke survivors. Moreover, the lack of appropriate inclusion criteria or objective tools to assess the treatment effects has been noted. Each acupuncture point

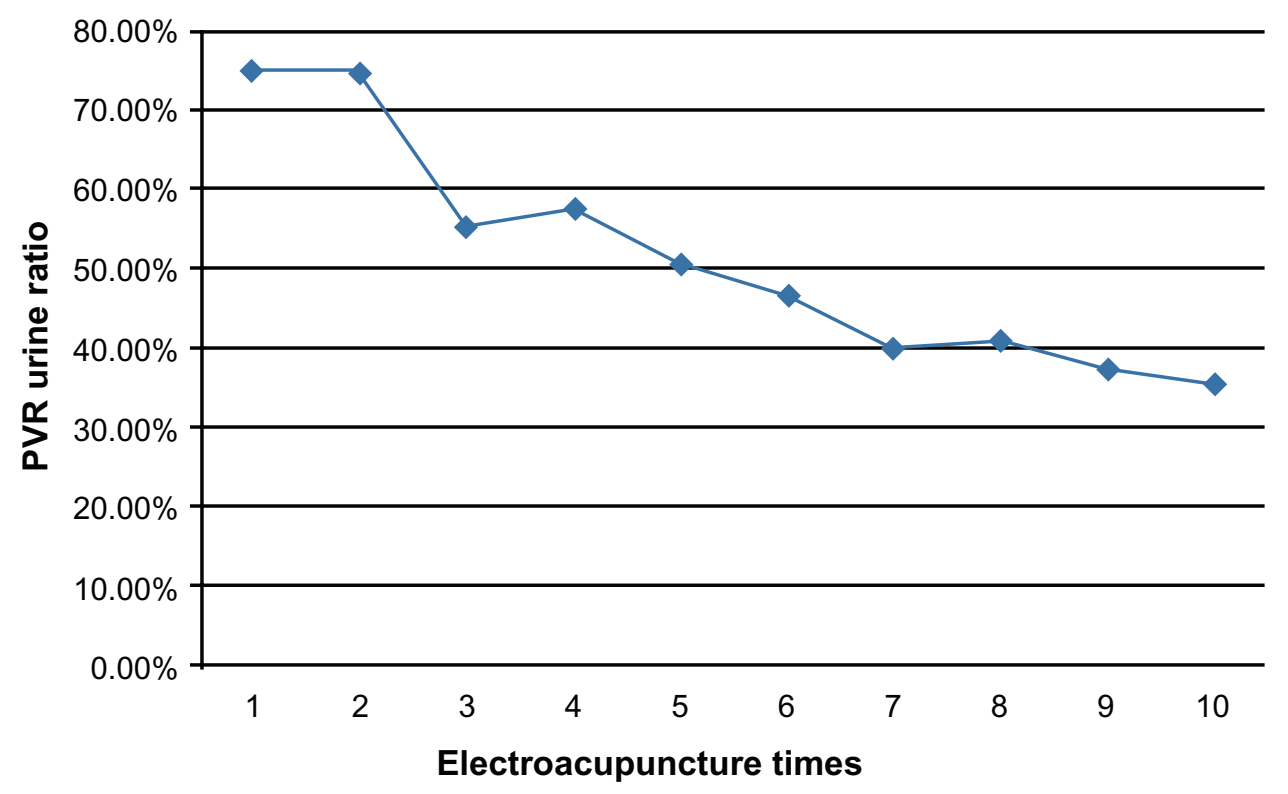

Figure 3 Arithmetic mean of the daily PVR urine ratio of all patients during the 10-day EA treatment.

Note: PVR urine ratio = (daily PVR urine volume/[daily PVR urine volume + spontaneous voiding volume]).

Abbreviations: PVR, postvoid residual; EA, electroacupuncture. 
has its distinct therapeutic actions. The acupuncture points used for EA treatment in this study, namely SP6, BL32, and BL28, improved the urinary function of patients.

Acupuncture could improve the voiding function by regulating the peripheral afferent nerve system. The locations of the two acupoints, BL28 and BL32, are similarly distributed along the S2-S4 dermatomes. The reflex voiding center lies at the S2 to S4 levels of the sacral spinal cord. BL28 and BL32 were stimulated by acupuncture to directly increase the excitability of the pelvic nerve, which consequently innervates the detrusor muscle. In addition, SP6 indirectly increases the excitability of the pelvic nerve. ${ }^{27,28}$ The increased detrusor pressure was previously observed after acupuncture treatment in animal experiments. ${ }^{28}$ The improvement in voiding function as an effect of acupuncture treatment has been proven by clinical studies. ${ }^{26,28-30}$

Either the mechanical action of an acupuncture needle or electrical point stimulation could achieve the appropriate stimulation of these acupuncture points. ${ }^{31}$

\section{Limitations of the study}

The present study was only a preliminary study with several limitations. First, the small sample size limited the statistical power for subgroup analyses. Second, no patient followup was conducted for an extended time period to observe whether the effects of EA treatment are sustained. Finally, there was no randomized control group with which to compare the natural course of bladder function improvement. Control groups are very important for comparisons among patients and for the assessment of treatment modalities. The limited case numbers and ethical considerations required the use of the pre-treatment conditions of patients as the controls, which were subsequently compared with their posttreatment conditions. Further studies with larger sample sizes and longer follow-up periods are required to confirm the findings of this preliminary study.

\section{Conclusion}

In our study, the effects of EA treatment on the subjective symptoms as well as the objective PVR urine conditions provided evidence that EA may be clinically useful in the management of post-stroke IBE. Further studies with larger sample sizes are required to confirm the findings of this study and to determine the sustainability of the effects of EA treatment.

\section{Disclosure}

Financial disclosure statements have been obtained, and no conflicts of interest have been reported by the authors or by any individuals in control of the content of this article. This study was supported by a grant from of the China Medical University Hospital (DMR-100107) and supported in part by Taiwan Department of Health Clinical Trial and Research Center of Excellence (DOH101-TD-B-111-004).

\section{References}

1. Brittain KR, Peet SM, Potter JF, Castleden CM. Prevalence and management of urinary incontinence in stroke survivors. Age Ageing. 1999;28(6):509-511.

2. Kong KH, Young S. Incidence and outcome of poststroke urinary retention: a prospective study. Arch Phys Med Rehabil. 2000;81(11): 1464-1467.

3. Frontera WR, editor. DeLisa's Physical Medicine and Rehabilitation. 5th ed. Philadelphia: Lippincott Williams \& Wilkins; 2011, chapter 23, page 569 .

4. Garrett VE, Scott JA, Costich J, Aubrey DL, Gross J. Bladder emptying assessment in stroke patients. Arch Phys Med Rehabil. 1989;70(1): 41-43.

5. Fowler CJ. Neurological disorders of micturition and their treatment Brain. 1999;122(Pt 7):1213-1231.

6. Hopwood V, Lewith GT. Does acupuncture help stroke patients become more independent? J Altern Complement Med. 2005;11(1):175-177.

7. Wu H, Tang J, Lin X, et al. Acupuncture for Stroke Rehabilitation. Stroke. January 3, 2008. [Epub ahead of print.]

8. Ling Z, Lin-bao G, Lian-fang C. Clinical study on early acupuncture for acuteischemic stroke. Journal of Acupuncture and Tuina Science. 2008;6:222-226.

9. Liu SY, Hsieh CL, Wei TS, Liu PT, Chang YJ, Li TC. Acupuncture stimulation improves balance function in stroke patients: a singleblinded controlled, randomized study. Am J Chin Med. 2009;37(3): 483-494.

10. Zhao JG, Cao CH, Liu CZ, et al. Effect of acupuncture treatment on spastic states of stroke patients. J Neurol Sci. 2009;276(1-2): 143-147.

11. Tang Y-Y, Lin C-H, Yu T-Y. Clinical evaluation in stroke patients for acupuncture and chinese manipulation combine with rehabilitation therapy. Journal of Chinese Medicine. 2010;21(1-2):53-61.

12. Ben $\mathrm{H}, \mathrm{Zu} \mathrm{Y,} \mathrm{Ye} \mathrm{Y.} \mathrm{The} \mathrm{effect} \mathrm{of} \mathrm{electroacupuncture} \mathrm{on} \mathrm{the} \mathrm{function}$ of the partially denervated bladder in rabbits. Zhen Ci Yan Jiu. 1993; 18(1):68-72. (Chinese).

13. Wang JH, Chen BG, Yin J, Wang G, Zou WG, Luo XJ. Effect of electroacupuncture of different acupoints on the excitability of detrusor muscle and the expression of BDNF and TrkB in the spinal cord of rats with urinary retention due to spinal cord injury. Zhen Ci Yan Jiu. 2009;34(6):387-392.

14. Yun SP, Jung WS, Park SU, et al. Effects of moxibustion on the recovery of post-stroke urinary symptoms. Am J Chin Med. 2007;35(6): 947-954.

15. Meng NH, Lo SF, Chou LW, Yang PY, Chang CH, Chou EC. Incomplete bladder emptying in patients with stroke: is detrusor external sphincter dyssynergia a potential cause? Arch Phys Med Rehabil. 2010;91(7): 1105-1109.

16. WHO Regional Office for the Western Pacific. WHO Standard Acupuncture Point Locations in the Western Pacific Region. Manila: World Health Organization; 2008:72,113,115.

17. Ireton RC, Krieger JN, Cardenas DD, et al. Bladder volume determination using a dedicated, portable ultrasound scanner. $J$ Urol. 1990;143(5):909-911.

18. Burney TL, Senapati M, Desai S, Choudhary ST, Badlani GH. Acute cerebrovascular accident and lower urinary tract dysfunction: a prospective correlation of the site of brain injury with urodynamic findings. J Urol. 1996;156(5):1748-1750. 
19. Maru A. Cystometry and urethral pressure profilometry after cerebral stroke. Nihon Hinyokika Gakkai Zasshi. 1980;71(2):171-183. (Japanese).

20. Arunabh M, Badlani. Urologic problems in cerebrovascular accidents. Problems in Urology. 1993;7(1):41-53.

21. Orikasa S, Imai Y, Igari D, et al. Urethral indwelling catheter, intermittent self-catheterization and urinary infection. Nihon Hinyokika Gakkai Zasshi. 1991;82(11):1807-1816. (Japanese).

22. Finkbeiner AE. Is bethanechol chloride clinically effective in promoting bladder emptying? A literature review. J Urol. 1985;134(3):443-449.

23. Lao L. Safety issues in acupuncture. J Altern Complement Med. 1996;2(1):27-31.

24. Kjendahl A, Sällström S, Osten PE, Stanghelle JK, Borchgrevink CF. A one year follow-up study on the effects of acupuncture in the treatment of stroke patients in the subacute stage: a randomized, controlled study. Clin Rehabil. 1997;11(3):192-200.

25. Lee SY, Baek YH, Park SU, et al. Intradermal acupuncture on shenmen and nei-kuan acupoints improves insomnia in stroke patients by reducing the sympathetic nervous activity: a randomized clinical trial. Am J Chin Med. 2009;37(6):1013-1021.
26. Tong Y, Jia Q, Sun Y, Hou Z, Wang Y. Acupuncture in the treatment of diabetic bladder dysfunction. JAltern Complement Med. 2009;15(8): 905-909.

27. Sato A, Sato Y, Suzuki A. Mechanism of the reflex inhibition of micturition contractions of the urinary bladder elicited by acupuncture-like stimulation in anesthetized rats. Neurosci Res. 1992; 15(3):189-198.

28. Yue-lai C, Lei Z. Progress of study on neural mechanism in acupuncture regulation of vesical functions. Acta Universitatis Traditionis Medicalis Sinensis Pharmacologiaeque Shanghai. 2002;16:63-65.

29. Yang DL. Acupuncture therapy in 49 cases of postpartum urinary retention. J Tradit Chin Med. 1985;5(1):26.

30. Cheng PT, Wong MK, Chang PL. A therapeutic trial of acupuncture in neurogenic bladder of spinal cord injured patients - a preliminary report. Spinal Cord. 1998;36(7):476-480.

31. Hurtak JJ. An overview of acupuncture medicine. JAltern Complement Med. 2002;8(5):535-538.
Clinical Interventions in Aging

\section{Publish your work in this journal}

Clinical Interventions in Aging is an international, peer-reviewed journal focusing on evidence-based reports on the value or lack thereof of treatments intended to prevent or delay the onset of maladaptive correlates of aging in human beings. This journal is indexed on PubMed Central, MedLine, the American Chemical Society's 'Chemical Abstracts Ser-

\section{Dovepress}

vice' (CAS), Scopus and the Elsevier Bibliographic databases. The manuscript management system is completely online and includes a very quick and fair peer-review system, which is all easy to use. Visit http://www.dovepress.com/testimonials.php to read real quotes from published authors. 